\title{
Local recurrence of hepatocellular carcinoma in the tumor blood drainage area following radiofrequency ablation
}

\author{
MASASHI HIROOKA ${ }^{1}$, HIRONORI OCHI ${ }^{1}$, YOHEI KOIZUMI ${ }^{1}$, YOSHIO TOKUMOTO ${ }^{1}$, \\ ATSUSHI HIRAOKA ${ }^{2}$, TERU KUMAGI ${ }^{1}$, MASANORI ABE ${ }^{1}$, HIROAKI TANAKA $^{3}$ and YOICHI HIASA ${ }^{1}$ \\ ${ }^{1}$ Department of Gastroenterology and Metabology, Ehime University Graduate School of Medicine, Toon, \\ Ehime 791-0295; ${ }^{2}$ Gastroenterology Center, Ehime Prefectural Central Hospital, Matsuyama, Ehime 790-0024; \\ ${ }^{3}$ Department of Radiology, Ehime University Graduate School of Medicine, Toon, Ehime 791-0295, Japan
}

Received August 14, 2013; Accepted November 25, 2013

DOI: $10.3892 / \mathrm{mco} .2013 .229$

\begin{abstract}
Hepatocellular carcinoma (HCC) is one of the most common malignant tumors worldwide. HCC is generally considered to spread via the bloodstream and local recurrence often occurs in the blood drainage area determined by computed tomography during hepatic arteriography (CTHA), despite complete ablation of the primary nodule. This study was conducted in order to prospectively assess the rate of local recurrence in the blood drainage area depicted by delayed-phase CTHA. The participants comprised 364 consecutive patients (260 men and 104 women; mean age, 67.4 \pm 8.6 years), enrolled between April, 2002 and December, 2011. The participants were divided into two groups, according to whether the ablation area covered the entire blood drainage area as defined by delayed-phase CTHA (group A) or not (group B). Local tumor progression was compared between the two groups. The median time to recurrence was significantly shorter for group B (434 days) compared to that for group A (1,474 days; $\mathrm{P}=0.0037)$. The cumulative local recurrence rates for group $\mathrm{A}$ were 0,0 and $1.5 \%$ at 1,3 and 5 years postoperatively, respectively, whereas the recurrence rates for group B were 3.8, 17.0 and $22.8 \%$ at 1,3 and 5 years, respectively $(\mathrm{P}<0.0001)$. In conclusion, the safety margin for radiofrequency ablation should be defined as the blood drainage area and ablation should aim at acquiring adequate safety margins.
\end{abstract}

Correspondence to: Professor Yoichi Hiasa, Department of Gastroenterology and Metabology, Ehime University Graduate School of Medicine, 454 Shizukawa, Toon, Ehime 791-0295, Japan E-mail: hiasa@m.ehime-u.ac.jp

Abbreviations: CTAP, computed tomography during arterial portography; CTHA, computed tomography during hepatic arteriography; ECOG, Eastern Cooperative Oncology Group; HCC, hepatocellular carcinoma; RFA, radiofrequency ablation; TACE, transcatheter arterial chemoembolization

Key words: blood drainage area, hepatocellular carcinoma, local recurrence, radiofrequency ablation

\section{Introduction}

Hepatocellular carcinoma (HCC) is one of the most common malignant tumors worldwide. Various therapeutic modalities are currently used to treat this disease, including liver transplantation (1), surgical resection $(2,3)$, percutaneous ethanol injection (4), radiofrequency ablation therapy (RFA) (5-7) and transcatheter arterial chemoembolization (TACE) (8). Liver cirrhosis with a low Child-Pugh grade due to infection with hepatitis virus is often present in HCC patients. Poor hepatic reserve function makes surgical resection difficult and liver transplantation is significantly limited by the shortage of organ donors. RFA has recently gained wide acceptance worldwide, mainly due to its relatively low level of invasiveness, ease of use and effectiveness $(9,10)$. The incidence of local tumor progression of HCC reportedly ranges between 0.9 and $31.3 \%(5,9,11-18)$. Local tumor recurrence may occur due to untreated satellite lesions, which are too small to detect on imaging prior to RFA (19). HCC is generally considered to spread via the bloodstream. A previous study by Sakon et al (20) reported that satellite lesions existed in the blood drainage area determined by computed tomography during hepatic arteriography (CTHA). In our experience, local recurrence often occurs in this blood drainage area, despite complete ablation of the primary nodule (Fig. 1). To the best of our knowledge, no prior study has evaluated the association between local recurrence following RFA and ablation of the blood drainage area.

The aim of this prospective cohort study was to evaluate the frequency of intrahepatic metastases within the blood drainage area of the tumor following RFA.

\section{Materials and methods}

Patients. All participants provided written informed consent prior to enrollment and the study protocols were approved by the Institutional Ethics Committee. The participants comprised 364 patients [260 men and 104 women; mean age \pm SD, $67.4 \pm 8.6$ years] who had been diagnosed with $\mathrm{HCC}$ at the Department of Gastroenterology and Metabology of Ehime University Hospital, Japan, between April, 2002 and December, 2011. The criteria for inclusion in the study were as follows: i) Eastern Cooperative Oncology Group (ECOG) 

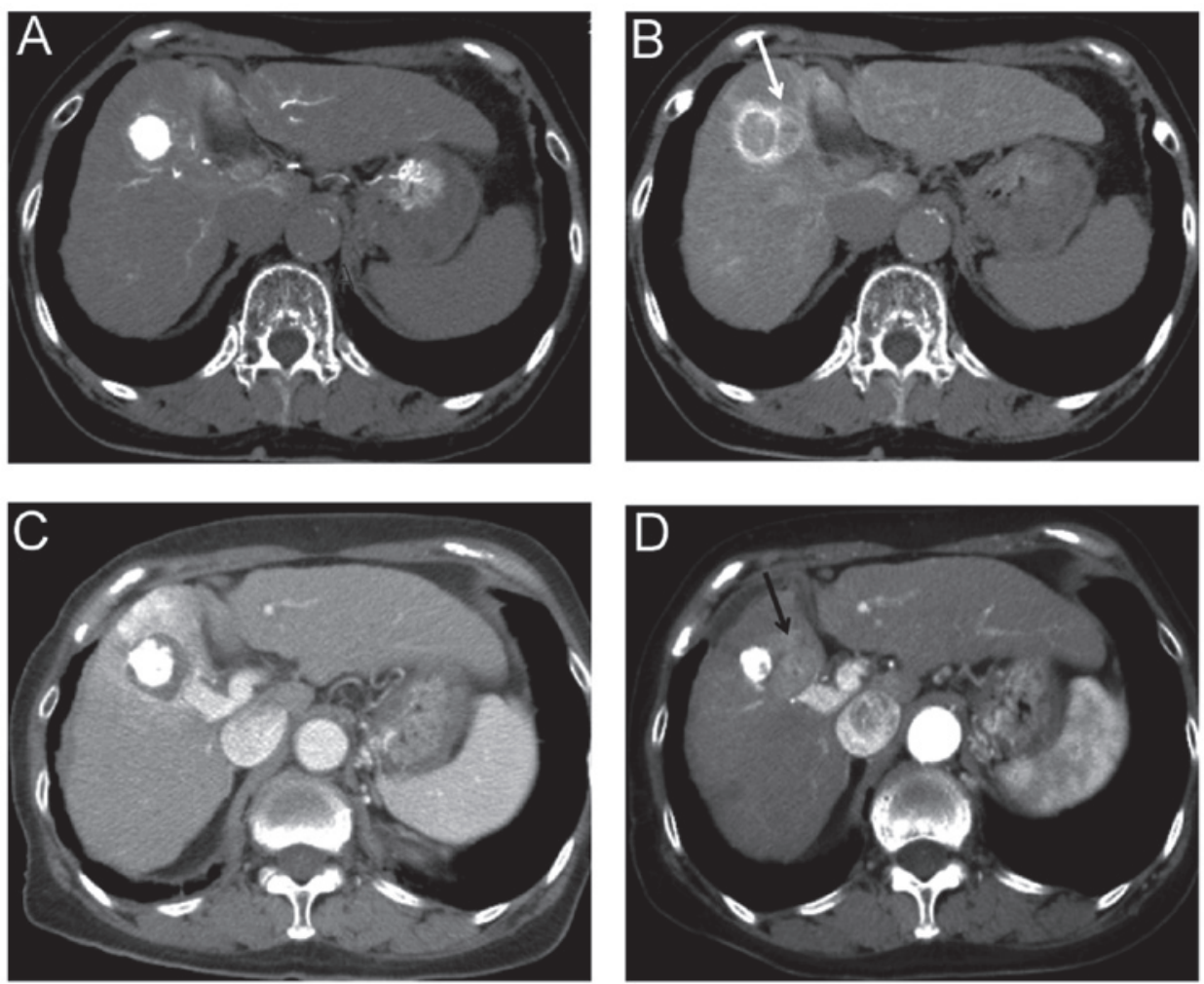

Figure 1. A case with local recurrence in the blood drainage area. (A) Early-phase computed tomography during hepatic arteriography (CTHA) in a 79-year-old female with hepatitis C-related liver cirrhosis (Child-Pugh class A) and hepatocellular carcinoma in S5. (B) Delayed-phase CTHA, reveals the blood drainage area around the tumor (white arrow). (C) Ablation was completed for the nodule itself. However, (D) local recurrence developed at a site within the blood drainage area depicted on delayed-phase CTHA (black arrow).

performance status $0-2$; ii) patients with unresectable lesions or who had declined hepatectomy; iii) presence of a single lesion $\leq 5 \mathrm{~cm}$ in diameter; iv) liver function A or B according to Child-Pugh classification; and v) no extrahepatic metastases or vascular invasion. The exclusion criteria were as follows: i) total bilirubin level $\geq 3.0 \mathrm{mg} / \mathrm{dl}$; ii) platelet count $<50,000 / \mathrm{mm}^{3}$; iii) prothrombin activity $<50 \%$; and iv) refractory ascites. HCC was defined as a nodule showing equivalent to decreased portal perfusion on computed tomography during arterial portography (CTAP) and increased arterial perfusion on early-phase CTHA with corona enhancement on late-phase CTHA. The combination of transarterial chemoembolization with RFA was performed for all the nodules.

RFA procedure and post-treatment analysis. RFA was performed with an internally cooled electrode (Radionics, Burlington, MA, USA). In each lesion, in order to obtain a suitable safety margin around the viable tumor, we selected a 2- or 3 -cm ablation-type needle. A total of 340 patients underwent ablation under ultrasonographic guidance $(6,7), 21$ patients using a laparoscopic procedure (21) and 3 patients by surgical incision. To assess the ablated area and complications, dynamic CT was performed within 3 days of the ablation. The goal of the treatment was to achieve complete ablation of the hypoattenuating areas visualized during the portal venous phases and extending beyond the tumor itself. Images from the portal venous phase were used, as the increase in inflammatory blood flow caused by the ablation could not be overlooked in the arterial phase. Additional ablation sessions were scheduled if the presence of residual lesions was confirmed. The diagnostic and treatment procedures were repeated until complete ablation was achieved during a single hospitalization.

CTHA. CTAP and CTHA were performed in all the patients prior to RFA, as previously described (22). A 4-Fr catheter was inserted through the femoral artery into the superior mesenteric artery for CTAP and into the common, proper, or replaced right hepatic artery for CTHA. CTHA scans were obtained at a section thickness and collimation of $3-5 \mathrm{~mm}$. Helical scanning was initiated $7 \mathrm{sec}$ after the infusion of iohexol (320-350 mg/ml of iodine) (Omnipaque; Daiichi, Tokyo, Japan) into the common, proper, or replaced hepatic artery at a rate of $1.8 \mathrm{ml} / \mathrm{sec}$. The infusion of contrast material was continued until $5 \mathrm{sec}$ after the completion of early-phase CTHA. The scanning time varied according to the individual liver size ( $20-25 \mathrm{sec})$. Thirty seconds after completing the contrast material infusion $(\sim 62-67 \mathrm{sec}$ after the initiation of the infusion), late-phase scanning was commenced (22). When a hypervascular tumor was identified on CTHA, the performance of transcatheter arterial embolization was considered mandatory prior to RFA, by injecting iodized oil (Lipiodol; Laboratoire Guerbet, Paris, France) with a gelatin sponge (Gelfoam; Upjohn, Kalamazoo, MI, USA) or gelatin sponge particles (Gelpart; Nippon Kayaku, Tokyo, Japan) into the segmental or subsegmental branch of the hepatic artery.

Imaging analysis. To assess the safety margins, the fusion imaging method was used. We used an open source DICOM 
Table I. Differences in clinical characteristics.

\begin{tabular}{|c|c|c|c|}
\hline Characteristics & $\begin{array}{c}\text { Uncovered } \\
\text { drainage } \\
\text { area }\end{array}$ & $\begin{array}{c}\text { Covered } \\
\text { drainage } \\
\text { area }\end{array}$ & P-value \\
\hline No. & 114 & 250 & - \\
\hline Female:male & $30: 84$ & $76: 174$ & 0.480 \\
\hline Age (years) & $67.2 \pm 8.5$ & $67.6 \pm 8.8$ & 0.695 \\
\hline Child-Pugh grade (A:B) & 104:10 & 198:52 & 0.539 \\
\hline $\begin{array}{l}\text { Etiology } \\
\text { (HBV:HCV:other) }\end{array}$ & 11:93:10 & 39:169:42 & 0.843 \\
\hline Tumor diameter (mm) & $21.9 \pm 8.1$ & $23.5 \pm 10.1$ & 0.127 \\
\hline $\begin{array}{l}\text { Width of ablated area } \\
(<5: 6-10:>10 \mathrm{~mm})\end{array}$ & 54:60:0 & $153: 65: 32$ & $<0.0001$ \\
\hline
\end{tabular}

$\mathrm{HBV}$, hepatitis B virus; $\mathrm{HCV}$, hepatitis $\mathrm{C}$ virus.

software (OsiriX Foundation; Geneva, Switzerland) capable of performing imaging fusion of $\mathrm{CT}$ images prior to and following RFA, as described previously (23). CT data were acquired from the CT workstation, with a $0.69-\mathrm{mm}$ thickness. One of two experienced radiologists performed CT image fusion using the manual segmentation registration method. The late phase of CTHA that depicted corona enhancement was selected and the portal phase of dynamic CT following RFA was fused to this image (Fig. 2).

Statistical analysis. Consecutive case series were enrolled in this study. Following ablation, the enrolled patients were divided into two groups, as follows: i) group A, ablated area covering the entire blood drainage area; or ii) group B, ablated area not covering the entire blood drainage area. The cumulative local recurrence rate was calculated using the Kaplan-Meier method and assessed using the log-rank test. Continuous parameters were expressed as median and range (10th and 90th percentiles) or as the means \pm SD. Statistical analysis was performed using the Student's t-test for unpaired data, the Mann-Whitney $\mathrm{U}$ test, $\chi^{2}$ test, Fisher's exact test, or Wilcoxon signed-ranks test, as applicable. $\mathrm{P}<0.05$ was considered to indicate a statistically significant difference. All data were analyzed using JMP software, version 9 (SAS Institute Japan, Tokyo, Japan).

\section{Results}

Clinical characteristics. The differences in the clinical characteristics between the two groups are shown in Table I. Except for the width of the ablation area, the baseline characteristics did not differ significantly between the two groups.

Recurrence. The cumulative local recurrence rates for all cases were 2.2, 6.7 and $9.9 \%$ at 1,2 and 3 years, respectively. Local recurrence was detected in 30 cases from the drainage area and in 6 cases from outside the drainage area (Fig. 3A). The median time to recurrence was 434 days (range, 170-959 days) for lesions in the drainage area and 1,474 days (range, 815-2,383 days) for lesions outside the drainage area $(\mathrm{P}=0.0037)$. Furthermore, the cumulative local recurrence rates were 0,0 and $1.5 \%$ at 1,3 and 5 years, respectively, for group $\mathrm{A}$ and $3.8,17.0$ and $22.8 \%$ at 1,3 and 5 years, respectively, for group B $(\mathrm{P}<0.0001$, Fig. 3B). As the width of the ablated margin increased, the local recurrence rates were significantly decreased $(\mathrm{P}<0.0001$, Fig. 4A). However, in cases without wide ablation margins $(<5 \mathrm{~mm})$, the local recurrence rates for group A remained significantly low $(\mathrm{P}=0.0017$; Fig. 4B). Among the cases in which sufficient ablation margins were achieved $(6-10 \mathrm{~mm})$, group A nodules also exhibited low rates of local recurrence $(\mathrm{P}=0.0022$; Fig. $4 \mathrm{C})$.

\section{Discussion}

In this study, the majority of local recurrences occurred within the blood drainage area depicted as the area of corona enhancement on CTHA. The prognosis for patients with local recurrence within this region was relatively poor. Therefore, treatment should be continued until the ablation area covers the entire blood drainage area. If an adequate safety margin cannot be achieved within the drainage area, a high risk of local recurrence must be anticipated.

HCC often exhibits satellite lesions $(19,24)$, which cannot be diagnosed by imaging, due to their small size. Previous studies reported satellite lesions located at a distance from the main nodule $(19,24)$. Various distances between the main and satellite nodules were previously reported; therefore, the ablated area may be determined based on those reports. Satellite lesions were found to be located $\leq 2 \mathrm{~mm}$ from the main nodule in $66.7 \%$ and $2.1-5 \mathrm{~mm}$ in $11.1 \%$ of the cases (24). Okusaka et al (19) reported that, of the 149 resected specimens, 28 (19\%) exhibited satellite lesions, which were located $\leq 0.5 \mathrm{~cm}$ from the main tumor in $8(33 \%), 0.6-1.0 \mathrm{~cm}$ in 12 (50\%), 1.1-1.5 $\mathrm{cm}$ in 1 (4\%) and 1.6-2.0 $\mathrm{cm}$ in $3(13 \%)$ of the cases. A safe margin was considered to be $\geq 5 \mathrm{~mm}(25)$. The present study also demonstrated significantly lower local recurrence rates for nodules ablated with wide safety margins (Fig. 4A). However, cases with safety margins $>5 \mathrm{~mm}$ but without ablation of the entire blood drainage area often developed local recurrence (Fig. 4C). Conversely, if a safety margin $>5 \mathrm{~mm}$ was not achieved, local recurrences were often not seen (Fig. 4B). Thus, the definition of safety margins should be determined using other factors in addition to distance from the primary lesion. Corona enhancement was recently proposed as reflecting the blood drainage area. Kitao et al (22) reported that intranodular capillarized sinusoids connect directly or indirectly to extranodular portal veins through portal venules within the fibrous septum. Sakon et al (20) reported that the high-risk area for intrahepatic metastases in the blood drainage area was confirmed from histopathological examination of the resected specimens. Satellite nodules in the drainage area were also reported as frequently being multiple moderately or poorly differentiated carcinomas, consistent with the characteristics of intrahepatic metastases. Furthermore, satellite nodules outside the drainage area were commonly solitary foci of well-differentiated carcinoma, suggestive of multicentric carcinogenesis. Those studies indicated that the surgical margins differed in each case with respect to tumor hemodynamics and, even in the same tumor, the width of safety margins differed according to the location of the tumor within the liver (20). The safety margin for RFA should be considered 

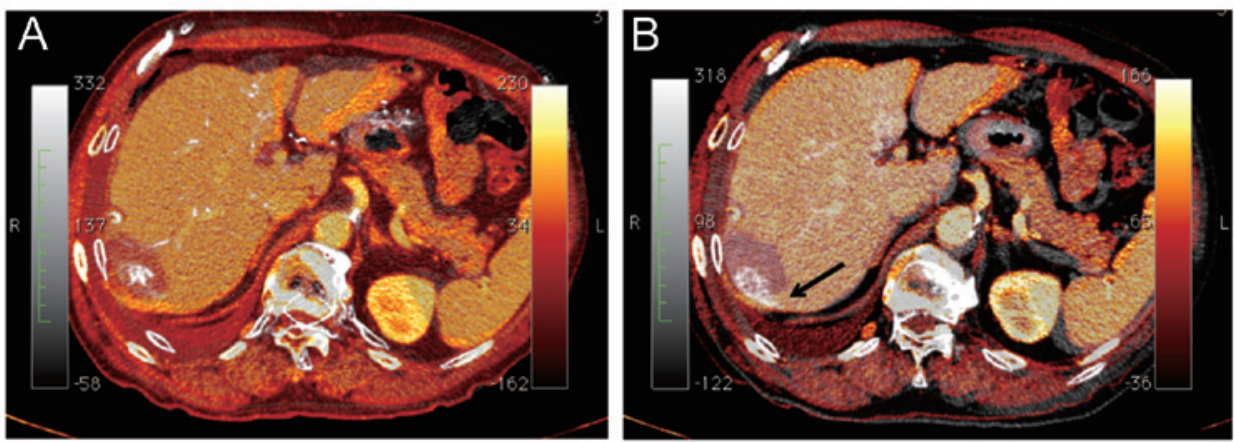

Figure 2. Analysis of ablation completeness. Overlay of computed tomography images obtained prior to ablation (gray scale) and following radiofrequency ablation therapy (RFA) (orange-coloured scale). (A) Computed tomography during hepatic arteriography (CTHA) prior to RFA is adopted as early-phase CTHA. The safety margin was considered to be sufficient. (B) Delayed-phase CTHA image depicting the blood drainage area was used for the gray scale image. The safety margin was inadequate (black arrow). The blood drainage area was not ablated in this case.
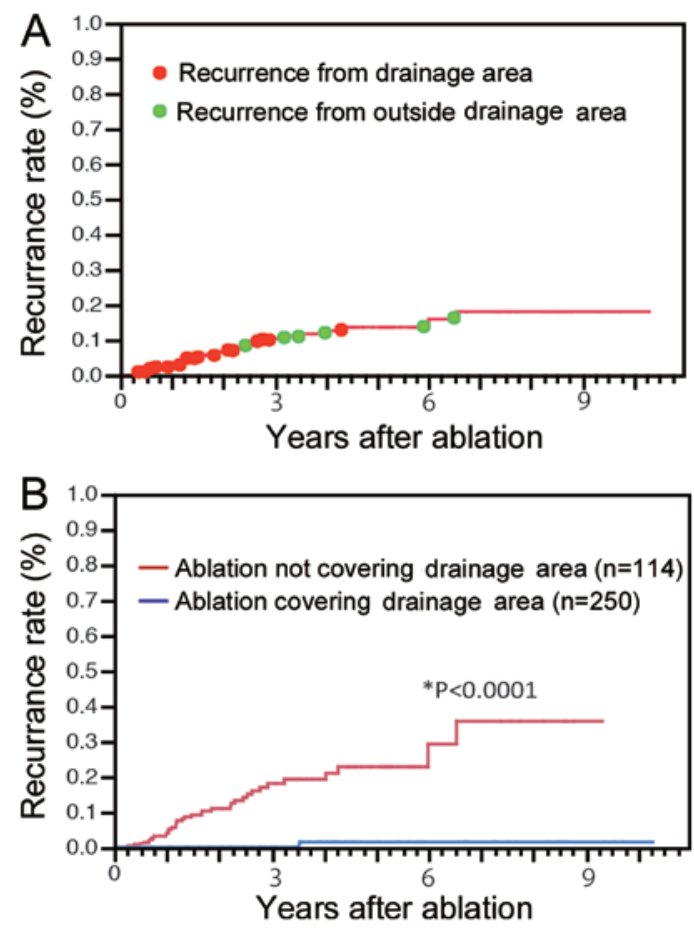

Figure 3. Overall local recurrence rate and ablation coverage. (A) Cumulative local recurrence rates for all cases were $2.2,6.7$ and $9.9 \%$ at 1,2 and 3 years, respectively. Local recurrence from the blood drainage area (red points) occurred significantly earlier following treatment compared to recurrence from outside the blood drainage area (green points). (B) The cumulative local recurrence rates for cases with ablation of the entire blood drainage area (blue line) were 0,0 and $1.5 \%$, respectively, compared to $3.8,17.0$ and $22.8 \%$, respectively, for cases without ablation of the entire drainage area (red line) $(\mathrm{P}<0.0001)$.

in the same way as the surgical margins for hepatectomy. Indeed, if the safety margin covered the entire blood drainage area, local recurrence was rarely reported in our study (Fig. 4).

Several limitations must be considered for this study. First, unlike a previous report (20), the lack of histological examination was problematic. Second, if the blood drainage area was $>10 \mathrm{~mm}$, obtaining safety margins was difficult. For example, for an HCC nodule diameter of $>30 \mathrm{~mm}$, an ablated area of $>50 \mathrm{~mm}$ should be obtained. In such cases, hepatectomy is warranted. If RFA is performed, TACE should be used as well. Third, delayed-phase CTHA was not performed at a single-slice level. Finally, fusion images are not absolutely
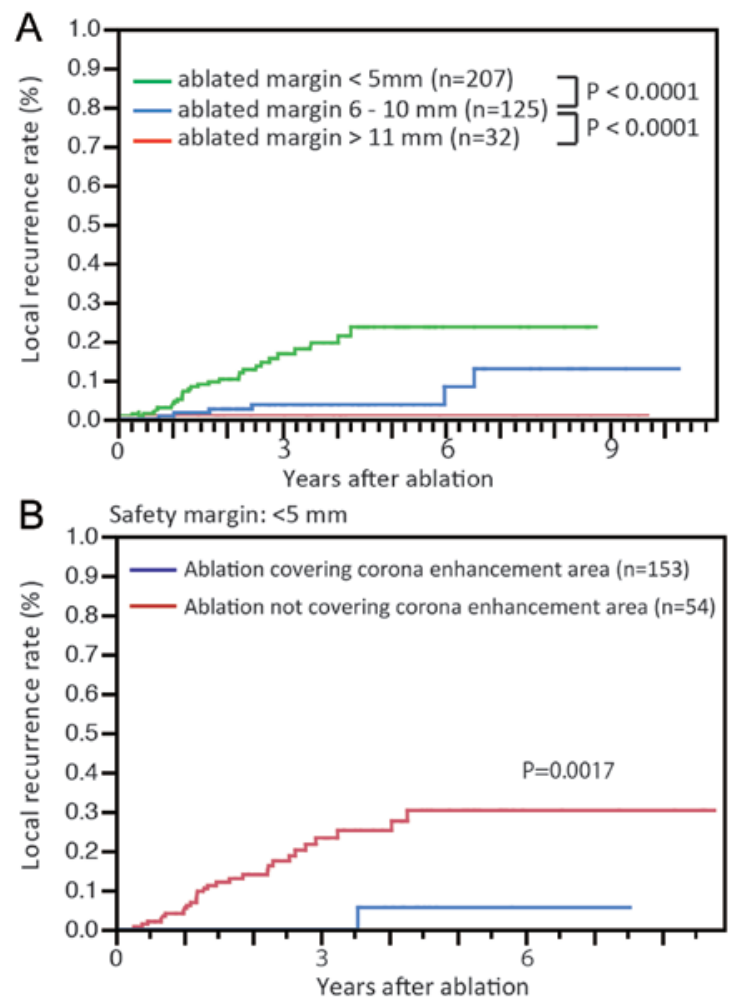

C ${ }_{1.0}$ Safety margin: $6-10 \mathrm{~mm}$

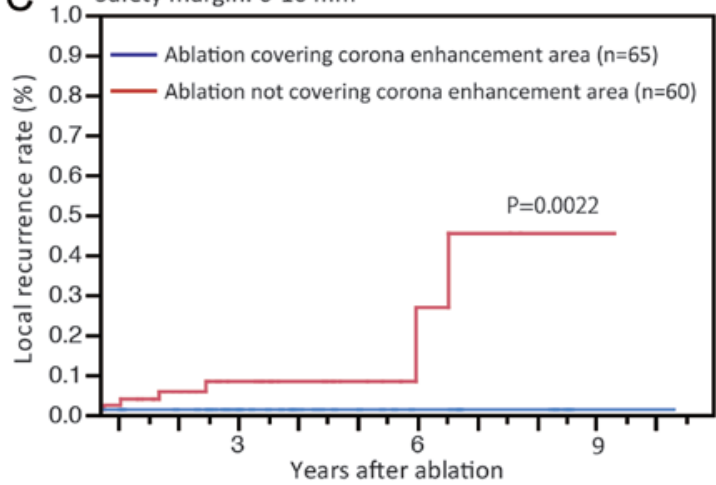

Figure 4. Local recurrence rates according to the width of the safety margin. (A) When the ablated margin was wide, the local recurrence rate was low. (B) For nodules with a narrow safety margin $(<5 \mathrm{~mm})$, local recurrence is rare if ablation covered the entire blood drainage area (blue line). (C) Local recurrence rates for ablation with or without coverage of the entire blood drainage area in patients with safety margins $6-10 \mathrm{~mm}$. Among the nodules with wide safety margins ( $>6 \mathrm{~mm})$, the risk of local recurrence was higher if ablation did not cover the entire blood drainage area (red line). 
accurate for assessing safety margins. A slight gap may be observed when images obtained on different days are overlapped.

In conclusion, the safety margins for RFA should be defined as the blood drainage area and ablation should be aimed at acquiring adequate safety margins.

\section{References}

1. Mazzaferro V, Regalia E, Doci R, et al: Liver transplantation for the treatment of small hepatocellular carcinomas in patients with cirrhosis. N Engl J Med 334: 693-699, 1996.

2. Arii S, Yamaoka Y, Futagawa S, et al: Results of surgical and nonsurgical treatment for small-sized hepatocellular carcinomas: a retrospective and nationwide survey in Japan. The Liver Cancer Study Group of Japan. Hepatology 32: 1224-1229, 2000.

3. Ikai I, Itai Y, Okita K, et al: Report of the 15th follow-up survey of primary liver cancer. Hepatol Res 28: 21-29, 2004.

4. Shiina S, Teratani T, Obi S, Hamamura K, Koike Y and Omata M: Percutaneous ethanol injection therapy for liver tumors. Eur J Ultrasound 13: 95-106, 2001

5. Shiina S, Teratani T, Obi S, et al: A randomized controlled trial of radiofrequency ablation with ethanol injection for small hepatocellular carcinoma. Gastroenterology 129: 122-130, 2005.

6. Uehara T, Hirooka M, Ishida K, et al: Percutaneous ultrasoundguided radiofrequency ablation of hepatocellular carcinoma with artificially induced pleural effusion and ascites. J Gastroenterol 42: 306-311, 2007.

7. Hirooka M, Iuchi H, Kumagi T, et al: Virtual sonographic radiofrequency ablation of hepatocellular carcinoma visualized on CT but not on conventional sonography. AJR Am J Roentgenol 186 (Suppl 5): S255-S260, 2006.

8. Takayasu K, Arii S, Ikai I, et al: Prospective cohort study of transarterial chemoembolization for unresectable hepatocellular carcinoma in 8510 patients. Gastroenterology 131: 461-469, 2006

9. Tateishi R, Shiina S, Teratani T, et al: Percutaneous radiofrequency ablation for hepatocellular carcinoma. An analysis of 1000 cases. Cancer 103: 1201-1209, 2005.

10. Hiraoka A, Horiike N, Yamashita Y, et al: Efficacy of radiofrequency ablation therapy compared to surgical resection in 164 patients in Japan with single hepatocellular carcinoma smaller than $3 \mathrm{~cm}$, along with report of complications. Hepatogastroenterology 55: 2171-2174, 2008.

11. Kim YS, Rhim H, Cho OK, Koh BH and Kim Y: Intrahepatic recurrence after percutaneous radiofrequency ablation of hepatocellular carcinoma: analysis of the pattern and risk factors. Eur J Radiol 59: 432-441, 2006.

12. Choi D, Lim HK, Rhim H, et al: Percutaneous radiofrequency ablation for early-stage hepatocellular carcinoma as a first-line treatment: long-term results and prognostic factors in a large single-institution series. Eur Radiol 17: 684-692, 2007.
13. Hori T, Nagata K, Hasuike S, et al: Risk factors for the local recurrence of hepatocellular carcinoma after a single session of percutaneous radiofrequency ablation. J Gastroenterol 38: 977-981, 2003.

14. Kim SH, Lim HK, Choi D, et al: Percutaneous radiofrequency ablation of hepatocellular carcinoma: effect of histologic grade on therapeutic results. AJR Am J Roentgenol 186 (Suppl 5): S327-S333, 2006.

15. Komorizono Y, Oketani M, Sako K, et al: Risk factors for local recurrence of small hepatocellular carcinoma tumors after a single session, single application of percutaneous radiof requency ablation. Cancer 97: 1253-1262, 2003.

16. Lencioni R, Cioni D, Crocetti L, et al: Early-stage hepatocellular carcinoma in patients with cirrhosis: long-term results of percutaneous image-guided radiofrequency ablation. Radiology 234: 961-967, 2005

17. Livraghi T, Meloni F, Di Stasi M, et al: Sustained complete response and complications rates after radiofrequency ablation of very early hepatocellular carcinoma in cirrhosis: is resection still the treatment of choice? Hepatology 47: 82-89, 2008.

18. Nakazawa T, Kokubu S, Shibuya A, et al: Radiofrequency ablation of hepatocellular carcinoma: correlation between local tumor progression after ablation and ablative margin. AJR Am J Roentgenol 188: 480-488, 2007.

19. Okusaka T, Okada S, Ueno H, et al: Satellite lesions in patients with small hepatocellular carcinoma with reference to clinicopathologic features. Cancer 95: 1931-1937, 2002.

20. Sakon M, Nagano H, Nakamori S, et al: Intrahepatic recurrences of hepatocellular carcinoma after hepatectomy: analysis based on tumor hemodynamics. Arch Surg 137: 94-99, 2002.

21. Hirooka M, Kisaka Y, Uehara T, et al: Efficacy of laparoscopic radiofrequency ablation for hepatocellular carcinoma compared to percutaneous radiofrequency ablation with artificial ascites. Dig Endosc 21: 82-86, 2009.

22. Kitao A, Zen Y, Matsui O, Gabata T and Nakanuma Y: Hepatocarcinogenesis: multistep changes of drainage vessels at CT during arterial portography and hepatic arteriography - radiologic-pathologic correlation. Radiology 252: 605-614, 2009

23. Kim YS, Lee WJ, Rhim H, Lim HK, Choi D and Lee JY: The minimal ablative margin of radiofrequency ablation of hepatocellular carcinoma $(>2$ and $<5 \mathrm{~cm})$ needed to prevent local tumor progression: 3D quantitative assessment using $\mathrm{CT}$ image fusion. AJR Am J Roentgenol 195: 758-765, 2010.

24. Nakashima Y, Nakashima O, Tanaka M, Okuda K, Nakashima M and Kojiro M: Portal vein invasion and intrahepatic micrometastasis in small hepatocellular carcinoma by gross type. Hepatol Res 26: 142-147, 2003.

25. Kudo M: Local ablation therapy for hepatocellular carcinoma: current status and future perspectives. J Gastroenterol 39 205-214, 2004. 